\title{
THE JUNE MEETING IN VANCOUVER
}

The four hundred thirty-seventh meeting of the American Mathematical Society was held at the University of British Columbia, Vancouver, Canada on Saturday, June 19, 1948. The attendance was approximately fifty, including the following 42 members of the society:

S. P. Avann, C. R. Ballantine, J. P. Ballantine, Samuel Beatty, R. A. Beaumont, E. F. Beckenbach, A. L. Blakers, Gertrude Blanch, J. L. Brenner, Daniel Buchanan, Albert Cahn, J. W. Campbell, W. B. Caton, C. M. Cramlet, Harold Davenport, Douglas Derry, S. P. Diliberto, D. G. Duncan, N. S. Free, W. H. Gage, J. W. Green, Olaf Helmer, M. G. Humphreys, R. D. James, S. A. Jennings, D. H. Lehmer, L. H. McFarlan, B. N. Moyls, D. C. Murdock, R. E. O'Connor, T. G. Ostrom, Edmund Pinney, Peter Scherk, W. H. Simons, Fritz Steinhardt, Otto Szász, Olga TausskyTodd, John Todd, W. L. G. Williams, Wilfrid Wilson, R. M. Winger, Clement Winston.

In the morning, there was a short session for research papers, at which Dean Daniel Buchanan presided. This was followed by the hour address, Recent progress in the Goldbach problem, by Professor R. D. James, of the University of British Columbia. Professor James was introduced by Professor Harold Davenport. In the afternoon, additional papers were presented in two sections, at which Professor R. M. Winger and Dean Samuel Beatty presided.

In the evening, those attending the meetings were the guests of the University of British Columbia at a dinner and social gathering at the student union. In addition, those who were fortunate enough to be able to spend several days in Vancouver were most hospitably entertained in many other ways by the University members and townspeople.

Abstracts of all papers presented at the meeting are given below. Papers read by title are indicated by the letter " $t$." Paper number 367 was presented by Mr. Todd, number 379 by Professor Jennings, and number 402 by Mr. Carter. Professor Volkoff and Mr. Carter were introduced by Professor R. D. James.

\section{Algebra and Theory of Numbers}

364t. Richard Bellman: On the number of squarefrees of the form $p+2$.

It is demonstrated that the number of squarefree numbers less than $x$ of the form $p+2$, where $p$ is a prime, is asymptotic to $c x / \log x$, where $c$ is a determined constant. The method also furnishes an error term. (Received April 29, 1948.)

365. J. L. Brenner: Equivalence of pencils of hermitian matrices under unitary transformations. Preliminary report. 
Necessary and sufficient conditions are obtained for the equivalence of pencils of hermitian matrices under unitary transformation. (Received June 18, 1948.)

\section{6t. Harold Chatland: On the Euclidean algorithm in quadratic number fields.}

The problem of determining whether or not the Euclidean algorithm exists in quadratic number fields has been worked out in all cases except for primes of the form $24 n+1$. L. Redei has shown that the algorithm exists in $R\left(73^{1 / 2}\right), R\left(97^{1 / 2}\right)$. Recently H. Davenport showed that the Euclidean algorithm does not exist for quadratic fields whose discriminant exceeds 16384 . In this paper, using a method due to Erdös and Ko, it is shown that for primes of the form $24 n+1$ greater than 97 the algorithm does not exist in $R\left(P^{1 / 2}\right)$ except possibly for $p=193,241,313,337,457,601$. (Received May 14, 1948.)

\section{S. D. Chowla and John Todd: The density of reducible integers.}

An integer $n$ is said to be reducible if the greatest prime factor of $1+n^{2}$ is less than $2 n$. It has been shown (John Todd, Amer. Math. Monthly, in the press) that reducibility of $n$ is a condition necessary and sufficient for the existence of a relation of the form $\arctan n=\sum f_{i} \arctan n_{i}$, where the $f_{i}$ are integers and the $n_{i}$ are positive integers less than $n$. It was noticed that the density of reducible integers (in the range $1 \leqq n$ $\leqq 5000$ ) was always very close to $.3 \ldots$. No result of this kind has yet been established but it has been shown that the density of those integers $n$ whose largest prime factor is less than $2 n^{1 / 2}$ is $1-\log 2=.3069 \ldots$ (Received May 11, 1948.)

\section{Harold Davenport: $A$ divisor problem.}

Many of the results proved in the analytic theory of numbers in the period up to 1937 can be improved by making use of the powerful inequalities for exponential sums devised by Vinogradov. As an example, consider the problem of the error term $R(x)$ in the formula $\sum_{n \leqq x} \sigma(n) / n=(1 / 6) \pi^{2} x-(1 / 2) \log x+R(x)$, where $\sigma(n)$ denotes the sum of the divisors of $n$. The classical estimate is $R(x)=O(\log x)$, and Walfisz (Math. Zeit. vol. 26 (1927) pp. 66-88) proved by means of Weyl's inequality that $R(x)=O[(\log x) /(\log \log x)]$. By using one of Vinogradov's inequalities, in the form given by Titchmarsh (Quart. J. Math. vol. 9 (1938) pp. 97-108), it is possible to prove that $R(x)=O(\log x)^{4 / 5+\epsilon}$ for any $\epsilon>0$. (Received May 17, 1948.)

\section{9t. W. M. Gilbert: Associative elements of quasi-groups.}

An element $a$ of a quasi-group $S$ is called left, center, or right associative if $a(x y)=(a x) y, x(a y)=(x a) y$, or $x(y a)=(x y) a$ for all $x$ and $y$ in S. G. N. Garrison (Ann. of Math. vol. 41 (1940) p. 479) has shown that in a commutative quasi-group left associative elements are right associative, and vice versa, and that left and right associative elements are center associative. An eighth order quasi-group is exhibited which shows that center associative elements need not be left or right associative. D. C. Murdoch has termed a quasi-group $S$ abelian if $(a b)(c d)=(a c)(b d)$, for all $a, b, c$, and $d$ in $S$. As a trivial extension of a lemma due to R. H. Bruck (Trans. Amer. Math. Soc. 55 (1944) p. 45), it is shown that if an abelian quasi-group contains a center associative element, or a left associative element and a right associative element, then it is an abelian group. (Received May 11, 1948.) 


\section{0t. Franklin Haimo: Subgroups with division properties in Abelian groups.}

Generalizations of the serving subgroups of Prüfer (Math. Zeit. vol. 20 (1924) pp. 165-187) and of the convex subgroups of Riss (C. R. (Doklady) Akad. Sci. URSS. N.S. vol. 13 (1947) pp. 987-988) are defined. If a subgroup has a generalized Riss property and a generalized Prüfer property of the types discussed, it is also convex. If a group $G$ contains a torsion-free cyclic subgroup with a generalized Prüfer property, then all the cyclic subgroups of $G$ have this property. A group is torsion-free if, and only if, all of its subgroups have some generalized Riss property or the convex property; and if a group contains a torsion-free subgroup with such a property, then the group itself is torsion-free. (Received May 14, 1948.)

\section{1t. J. L. Hodges and Alfred Horn: On Maharam's conditions for} measure.

In a recent paper (Ann. of Math. vol. 48 (1947) pp. 154-167), Dorothy Maharam gave a set of necessary and sufficient conditions that a countably complete Boolean algebra have a strictly positive, countably additive measure. The purpose of this note is to point out that one of these conditions (Condition I) is a consequence of two of the others (Conditions II and IIIa). The proof uses Theorem 2 of Maharam's paper. (Received May 14, 1948.)

372t. Eri Jabotinsky: The arithmetical properties of the coefficients $A_{n}$ in the expansion $\left(e^{z}-1\right) /\left(e^{z}-z\right)=\sum_{0}^{\infty} A_{n} z^{n} / n !$.

The $A_{n}$ satisfy: $A_{0}=0$ and $1+A_{n-1}=\sum_{k=0}^{n}\left(C_{n, k}\right) A_{k}$, and $A_{n}$ is integral. Explicitly: $A_{n}=(n+1) ! \sum_{k=0}^{n}(-n-1+k)^{(k-1)} / k !, n>0$. Therefore, $A_{n} \equiv(-1)^{n-1} \bmod n$ and for $n>0$, no $A_{n}$ is 0 . Also $A_{n} \equiv-n ! \bmod (n+1)$; whence, by Wilson's theorem, $A_{n} \equiv 1 \bmod$ $(n+1)$ if and only if $(n+1)$ is prime. If $n-m=p$ is prime, then, by Fermat's theorem, $A_{n} \equiv A_{m}^{\prime} \bmod p$, where $A_{m}^{\prime}=(m+1) ! \sum_{k=0}^{m}(-m-1+k)^{k} / k !$. More generally, for integral $l \geqq 0$, let $A_{m}^{l}=(m+1) ! \sum_{k=0}^{m}(-m-1+k)^{(l-1+k)} / k !$. The $A_{m}^{l}$ are integral and $A_{m}^{l+1}=(m+1)$ - $\left(A_{m-1}^{l+1}-A_{m}^{l}\right)$ if $n-m=p$ is prime, then $A_{n}^{l} \equiv A_{m}^{l+1} \bmod (n-m)$. Hence $A_{n}=A_{n}^{0}$ $\equiv A_{n-p l}^{l n} \bmod p$. Examples: for $m \geqq 1, A_{2 m} \equiv 1 \bmod 2$ and $A_{2 m+1} \equiv 0 \bmod 2$, and for $m \geqq 2$, $A_{3 m-1} \equiv 0 \bmod 3, A_{3 m} \equiv(-1)^{m-1} \bmod 3$, and $A_{3 m+1} \equiv-1+(-1)^{m-1} \bmod 3$. Also $\sum_{k=0}^{l}(-1)^{k}\left(C_{l, k}\right) A_{l-k}^{k}=0$, so that for prime $p, \sum_{k=0}^{l}(-1)^{k}\left(C_{k, l}\right) A_{l+k(p-1)} \equiv 0 \bmod p$. Consider the function $F(w, z)=\left(e^{z}-w\right) /\left(e^{z}-w z\right)$ and the operator $\alpha=-w(d / d w)$. Then $\alpha^{l} F(1, z)=\sum_{n-0}^{l} A_{n}^{\infty} z^{n} / n !$. Let $\sum b^{-n}$ designate the sum over all roots $b$ of the equation $b=e^{b}$. Then $\sum b^{-n}=-A_{n-1}$, which permits to extend the definition of $A_{n}$ to all real $n$ greater than zero. (Received April 20, 1948.)

\section{D. H. Lehmer: Six multiplicative functions associated with} Ramanujan's $\tau(n)$.

Let $\sigma_{k}(n)$ denote the sum of the $k$ th powers of the divisors of $n$ and let $\tau(n)=\tau_{1}(n)$ be the coefficient of $x^{n-1}$ in the expansion of the 24th power of the product $(1-x)\left(1-x^{2}\right)\left(1-x^{3}\right) \ldots$. We consider six functions $\tau_{\lambda}(n)$ defined for $\lambda=1,2, \cdots, 6$ by $\tau_{\lambda}(n)=\tau_{1}(n)+A \sum_{m=1}^{n} \sigma_{n}(m) \tau_{1}(n-m)$ where $(A, h)=\left(A_{\lambda}, h_{\lambda}\right)$ have the six values $(0,-1),(240,3),(-504,5),(480,7),(-264,9)$ and $(-24,13)$. These functions, already noticed by Ramanujan and Hecke, are of interest in connection with a question recently raised by $S$. Chowla concerning the sum $\sum \sigma_{1}\left(a_{1}\right) \sigma_{1}\left(a_{2}\right) \cdots \sigma_{1}\left(a_{m}\right)$ extending over all positive integers $a_{i}$ whose sum is $n$. Besides the functions $\sigma_{k}(n)$, these six are 
the only multiplicative functions occurring in the expression for this sum. They all have properties similar to those of Ramanujan's $\tau_{1}(n)$. For $n=p^{\alpha}, p$ a fixed prime, $\tau(n)$ form a recurring series of the second order whose scale is $x^{2}-\tau_{\lambda}(p) x+p^{h+12}$. There are numerous congruence properties. For example $\tau_{\lambda}(n)$ is congruent to $\sigma_{h+18}(n)$ modulo the numerator of the $(h+13)$ th Bernoulli number. The Fourier series, Dirichlet series, and certain Bessel series involving $K_{v}(x)$, whose coefficients are $\tau_{\lambda}(n)$, all have characteristic properties. (Received May 13, 1948.)

\section{B. N. Moyls: The structure of valuations of the rational function} field $K(x)$.

Let $V_{0}$ be a rank one valuation of a field $K$ with value group $G_{0}$ and residue class field $F_{0}$; and let $V$ be a rank one extension of $V_{0}$ to the field $K(x)$ with value group $G$ and residue field $F$. By a method due to S. MacLane (cf. Trans. Amer. Math. Soc. vol. 40 (1936) pp. 363-395, for a treatment of the case in which $V_{0}$ is discrete) it is shown that the sum of the transcendence degree $T\left[F / F_{0}\right]$ of $F$ over $F_{0}$ and the rational rank of the factor group $G / G_{0}$ must be less than or equal to one. If the equality holds, $F$ and $G$ can be finitely generated over $F_{0}$ and $G_{0}$, respectively. If $T\left[F / F_{0}\right]=1, F$ is a rational function field over a finite algebraic extension of $F_{0}$. In every case, $F$ and $G$ are at most denumerable extensions of $F_{0}$ and $G_{0}$, respectively. Conversely it is shown that extensions of $V_{0}$ to $K(x)$ with prescribed value groups and residue class fields conforming to the above restrictions can be constructed in all cases except perhaps where $G / G_{0}$ is finite and $F$ is a finite algebraic extension of $F_{0}$. (Received May 13, 1948.)

\section{Ivan Niven: On the location of the roots of a polynomial and its derivative.}

It is proved that, for a polynomial with real roots, the average distance between the roots is not less than the corresponding average for the derivative. It has been conjectured by Paul Erdös and the writer that this result holds for any polynomial with complex coefficients. (Received May 17, 1948.)

376t. H. E. Salzer: Table expressing every square up to one million as a sum of four non-negative tetrahedral numbers.

The author's empirical theorem that every square integer is the sum of four nonnegative tetrahedral numbers, $n(n+1)(n+2) / 6, n \geqq 0$ (whose verification was announced previously for the first 300 squares), has now been verified as far as the first 1000 squares. A manuscript table establishing this fact is in the author's possession. Thus to disprove this conjecture would necessitate finding a square greater than $1,000,000$ (that is, after the 1000th case) which requires five tetrahedral numbers. (Received April 9, 1948.)

\section{W. H. Simons: Modular functions of stufe 2.}

Convergent series are obtained for the Fourier coefficients of $\kappa^{2}=\lambda(\tau)$, the fundamental modular invariant of stufe 2, and also for the reciprocal of $\lambda(\tau)$. Moreover, the series obtained for the Fourier coefficients of $\lambda(\tau)$ and $\mu(\tau)=1 / \lambda(\tau)$ are subseries of that obtained by Rademacher for Klein's absolute invariant $J(\tau)$. The terms whose subscripts are of the form $4 n+2$ in the series for the Fourier coefficients of $J(\tau)$ give 
the terms of the series for $\lambda(\tau)$, while the terms whose subscripts are of the form $4 n$ give those for $\mu(\tau)$. (Received May 13, 1948.)

\section{Olga Taussky: Bounds for characteristic roots of matrices.}

A. Brauer (Duke Math. J. vol. 13 (1946)) proved that all the characteristic roots of the $n \times n$ matrix $\left(a_{i j}\right)$ with complex elements lie inside or on the boundary of the circles with centres $a_{i i}$ and radii $\sum_{j \neq i}\left|a_{i j}\right|$. Several generalizations are made; for example, the boundary points of the domain formed by the circles cannot, in general, be roots. If $n=2$, inner points in common to both circles cannot be roots. Results concerning multiple roots are obtained. (Received May 13, 1948.)

379. R. M. Thrall and S. A. Jennings: Some remarks on quasi-cyclic algebras.

In a recent paper (Bull. Amer. Math. Soc. 53 (1947) pp. 369-377) G. Hochschild has introduced the notion of quasi-cyclic algebra, and has shown that to every separable algebra $B$ with radical $R \neq 0$ there exists a unique maximal quasi-cyclic extension. The present note is concerned with some of the properties of quasi-cyclic algebras. In particular, examples are given to show that no minimal quasi-cyclic extension exists in general. (Received May 15, 1948.)

\section{ANALYsis}

380. E. F. Beckenbach: On harmonic, subharmonic, and linear functions of several variables.

Equalities and inequalities between area and circumferential means of positive functions are investigated. It is shown, for instance, that if $f(x, y)$ is positive and of class $C^{\prime \prime}$ in a domain $D$, then its harmonic area mean is equal to its geometric circumferential mean for all circular discs in $D$ if and only if $f(x, y)$ is a linear function. (Received May 15, 1948.)

381t. Richard Bellman: On analytic point transformations and interpolation of iterates.

It is shown that if $f(x)=\sum a_{n} x^{n}, 1 \leqq n<\infty$, is an analytic point transformation satisfying the conditions that $\sum\left|a_{n}\right|<1, a_{1} \neq 0$, then $f(x)$ is the value at $t=1$ of the solution of an analytic differential equation of the form $y^{\prime}=\sum b_{n} y^{n}, 1 \leqq n<\infty, y(0)=x$, provided that $|x|$ is sufficiently small. This shows that $f(x)$ and its iterates may be imbedded in a continuous semi-group of functions, $f(x, t)$, having the properties that $f(f(x, t), s)=f(x, s+t)$, and that $f(x, n)$ is the $n$th iterate of $f(x)$, for $s$ and $t \geqq 0$. (Received April 29, 1948.)

382t. Richard Bellman: On the asymptotic behavior of solutions of linear differential equations. Preliminary report.

The behavior of solutions of the differential equations, $a(t) y^{\prime \prime}+y^{\prime}+y=0$, $y^{\prime \prime}+b(t) y^{\prime}+y=0$, is studied as $t \rightarrow+\infty$, under the assumptions that $a(t) \rightarrow 0$ and $b(t) \rightarrow+\infty$ as $t \rightarrow+\infty$. It is shown that all solutions of the second differential equation do not necessarily $\rightarrow 0$ as $t \rightarrow+\infty$. (Received April 29, 1948.) 
383t. Richard Bellman: On the summability of solutions of linear integral equations.

The summability of the Liouville-Neumann solution of the Fredholm integral equation is studied. It is shown that for the case of a continuous symmetric kernel, the Liouville-Neumann solution is summable to the Fredholm solution for all values of the parameter not equal to characteristic values by any summation method which sums the series $\sum z^{n}$ to $1 /(1-z)$ for all values of $z \neq 1$. Other methods of summabilityarealso discussed. (Received April 29, 1948.)

\section{W. B. Caton: On asymptotic formulas for Laguerre polynomials.}

In this note the general asymptotic methods of Langer are used to obtain asymptotic formulas of the Plancherel-Rotach type for Laguerre polynomials. (Received May 4, 1948.)

\section{5t. Harvey Cohn: Diophantine aspects of modular functions. I.}

The author investigates the relationship between modular functions and real irrationals by considering the behavior of certain modular functions at their singularities. This process must reveal properties of real numbers invariant under the modular group $z^{\prime}=(a z+b) /(c z+d)$, where $a, b, c, d$ are integers which satisfy the condition $a d-b c=1$. For instance, when the complex variable $z$ approaches the rational number $p / q$ in a Stolz (angular) region, the Eisenstein series $\sum(a z+b)^{-2 \alpha}$ behaves, as expected from the individual terms, like const. $\cdot(z-p / q)^{-2 \alpha}$. Likewise, this series of the Poincare theta type: $G_{2 \alpha}(z)=\sum\left(c z^{2}+(a+d) z+b\right)^{-2 \alpha}$, summed over $a d-b c=1$, behaves like $O(z-\theta)^{-2 \alpha}$ as $z \rightarrow \theta$ in a Stolz region at $\theta$, where $\theta$ is a quadratic irrational, or more generally, any irrational number with bounded partial denominators in its simple continued fraction. It is further shown that $G_{12}(z)$ accounts for all Poincare theta functions for which $(z-\theta)^{-2 \alpha}$ is the exact order of magnitude of irrational singularities with bounded denominators, approached in any Stolz region. The method used is to express these functions in terms of the elliptic modular function and to keep transforming the plane as $z$ approaches $\theta$, so as to magnify the region about the point $\theta$. (Received March 19, 1948.)

\section{C. M. Cramlet: $A$ generalization of a theorem of Jacobi.}

A system of $k$ contravariant vectors $A_{(i)}, i=1,2, \cdots, k, \alpha=1,2, \cdots, n$, is said to be Jacobian if the associated contravariant vectors $T_{(i j)}^{\beta} \equiv A_{(i)}^{\alpha} \partial A_{(j)}^{\beta} / \partial x^{\alpha}$ $-A_{(i)}^{\alpha} \partial A_{(i)}^{\beta} / \partial x^{\alpha}$ vanish identically. For such systems a class of multipliers $M$ exist, which are relative invariants of weight one and have the property that the tensor densities $\partial\left(M A_{(i)}^{\alpha}\right) / \partial x^{\alpha}$ vanish. In particular, when $k=n-1$ and $\lambda_{\alpha}$ is the vector product of the $n-1$ vectors, $M \lambda_{\alpha}$ is a gradient. The contravariant vectors determine a Jacobian system of $k$ linear partial differential equations, the covariant vector, an associated Pfaffian with $M$ as its integrating factor. (Received June 9, 1948.)

387t. R. F. Deniston: Existence of integrals in the Pollard-Moore sense. Preliminary report.

Let $N$ and $\sigma$ denote for integrals the norm and Pollard-Moore sense respectively, following the notation of Hildebrandt (Amer. Math. Monthly vol. 45 (1938) pp. 265278). The Cauchy left $\sigma$-integral, to be denoted by $\sigma^{(-)} \int_{a}^{b} f d g$, has been studied for the case $f$ bounded with only simple discontinuities and $g$ nondecreasing in $\langle a, b\rangle$ by 
Price (Bull. Amer. Math. Soc. vol. 49 (1943) pp. 625-630). The following theorems give conditions for the existence of the specified integrals for the case of $f$ bounded, $g$ of bounded variation in $\langle a, b\rangle$. In Theorems 1-3 conditions given are necessary and sufficient. Theorem 1 . For the existence of $\sigma^{(-)} \int_{a}^{b} f d g$ : (a). In every point of the interval $(a, b)$ in which $g$ is discontinuous on the left the function $f$ has a left limit, $f(x-0)$. $(b)$. The set of the points in $(a, b)$ which are left-sided discontinuities of the function $f$ is a null set with respect to the continuity function of $g$. (A similar theorem holds for the $\sigma$-right Cauchy integral.) Theorem 2. The condition that the interval function on which $\sigma^{(-)} \int_{a}^{b} f d g$ is based be pseudo-additive in the sense of Getchell is that in every point of $(a, b)$ in which $g$ is discontinuous on the right the function $f$ be continuous on the left. Getchell (Bull. Amer. Math. Soc. vol. 49 (1943) pp. 413-418) has given a theorem which together with Theorems 1 and 2 proves Theorem 3 . For the existence of $N^{(-)} \int_{a}^{b} f d g:\left(\mathrm{a}^{\prime}\right)$. Same as $(\mathrm{a}) ;\left(\mathrm{b}^{\prime}\right)$. The set of the points in $(a, b)$ which are left-sided discontinuities of the function $f$ is a null set with respect to the left-sided continuity function $g_{l}$ of $g$. This theorem has been given by Schaerf (Portugaliae Mathematica vol. 4 (1943-1944) pp. 73-118). (Received May 14, 1948.)

388t. P. R. Garabedian: Distortion of length in conformal mapping. Preliminary report.

Let $D$ be a domain of the $z$-plane of finite connectivity bounded by analytic curves $C$. A method is developed for finding extremal functions $f_{0}(z)$ minimizing the length integral $\int_{C}\left|f^{\prime}(z)\right||d z|$ for various different normalizations of the conformal mappings $f(z)$. The idea consists in solving extremal problems of the Schwarz lemma type in the normal, compact class of functions $F(z)$ mapping each countour of $C$ into a countour of diameter not greater than 2. One poses such a problem in the subclass of maps on Riemann surfaces $S$ bounded by non-concentric unit circles. A method of variation of the branch points of the extremal surface $S_{0}$ is developed which leads to a quadratic differential $R(z) d z^{2}$ with zeros at the branch points of $S_{0}$. Let $F_{0}$ be the map of $D$ on $S_{0}$. Then the relation $f_{0}^{\prime}(z) F_{0}^{\prime}(z) d z^{2}=R(z) d z^{2}$ yields the extremal function $f_{0}(z)$ for the minimum length problem. The proof of extremal properties is carried out by the method of contour integration, using the relation $R(z) d z^{2} \leqq 0$ on $C$. Variational formulas of the Schiffer type are developed for the branch point variations, and the results are generally applicable throughout conformal mapping. Loewner's differential equation can be derived as a special case. (Received May 6, 1948.)

\section{9t. M. O. Gonzalez: On a certain class of transcendental func- tions.

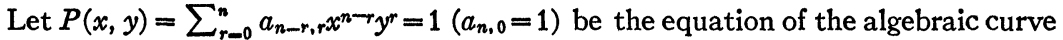
$C$. Let $u=2 \cdot$ area $O A M$, where $O$ is the origin, $A \equiv(1,0)$, and $M \equiv(x, y)$ a point on the branch of $C$ through $A$. Then by definition $\operatorname{Cos} u=x, \operatorname{Sin} u=y, \operatorname{Tan} u=y / x$, and so on. Some general properties of these generalized trigonometric functions are proved, in particular, the differentiation formulas $D(\operatorname{Sin} u)=P_{x} / n, D(\operatorname{Cos} u)=-P_{y} / n, D(\operatorname{Tan} u)$ $=1 / \cos ^{2} u=\sec ^{2} u$. The author considers in detail the case $P(x, y)=x^{4}+2 \lambda x^{2} y^{2}$ $+y^{4}=1$, for which $D(\operatorname{Sin} u)=\operatorname{Cos} u\left(\operatorname{Cos}^{2} u+\lambda \operatorname{Sin}^{2} u\right), D(\operatorname{Cos} u)=-\operatorname{Sin} u\left(\lambda \operatorname{Cos}^{2} u\right.$ $\left.+\operatorname{Sin}^{2} u\right)$, thus furnishing a comprehensive account of the circular $(\lambda=1)$, hyperbolic $(\lambda=-1)$ and elliptic functions $(|\lambda| \neq 1)$. The addition theorems, duplication formulas, and so on, and several formulas connecting Tan $u$ with the elliptic functions of Jacobi and Weierstrass are given. For complex values of the argument Tan $u$ is defined by the 
formula Tan $u=\vartheta_{1}(v) \vartheta_{3}(v) / \vartheta_{0}(v) \vartheta_{2}(v), u=\pi v \vartheta_{z}^{2}$, and its zeros, poles, periods, expansions and other properties are studied. (Received May 11, 1948.)

\section{0t. J. P. LaSalle: Uniqueness theorems and successive approxi- mations.}

Two cases where the method of successive approximations provides a sequence of functions which converge to a solution of $(1) y_{i}^{\prime}=f_{i}\left(t, y_{1}, \cdots, y_{n}\right), i=1, \cdots, n$, are considered. In the first case the solution need not be unique but by starting with suitable zero approximations the method applies. A theorem which gives bounds on the solutions of (1) is obtained and leads to a uniqueness theorem which includes those of Osgood, Montel, and Nagumo. Under the conditions of this theorem the solution can be successively approximated. The interval of convergence and the class of zero approximations are as in Picard's classical theorem with the Lipschitz condition. This is an extension of Wintner's recent result for Osgood's uniqueness condition and of a much earlier theorem by A. Rosenblatt (1909). (Received April 27, 1948.)

\section{Otto Szász: Summation of slowly convergent series.}

Given a series $\sum_{0}^{\infty} U_{n}$ or the sequence of its partial sums $s_{n}=\sum_{\nu=0}^{n} U_{\nu}$; the author considers transforms $t_{n}$ which yield more rapid convergence for certain classes of series, such as convergent series with positive or alternating terms. A case in point is the transform $t_{n}=s_{n} \tan U_{n}$, where $a$ is a constant. If for instance $U_{n}>0$ and for some constant $b>1, U_{n} / U_{n-1}=1-b / n+O\left(n^{-2}\right)$, then the best choice for $a$ is $a=1 /(b-1)$. The subclass of series with positive terms for which $t_{n}$ converges more rapidly than $s_{n}$ can be characterized, and $s-t_{n}$ can be estimated. For $a=1, t_{n}$ is the inverse of the $(C, 1)$ transform: $s_{n}=\left(\sum_{0}^{n} t_{v}\right) /(n+1)$. Discussion of other transforms and their inverses, such as Euler's method, leads to further results. Some applications to numerical series are given. (Received May 13, 1948.)

\section{2t. Cengiz Uluçay: On the values of the Bloch-Landau constants} $\mathfrak{B}, \mathfrak{R}$. I.

Fundamental Lemma I. Let $G$ be the total group of the inner automorphisms $g$ which leave fixed the unit circle $|z|<1$. Let $R$ be the fundamental region corresponding to the properly discontinuous subgroup $H \subset G$. Consider all the conjugate subgroups $\mathrm{gHg}^{-1}, \mathrm{~g} \notin H$. Then there exists a one-to-one continuous correspondence between the totality of the elements $\left\{\mathrm{gHg}^{-1}\right\}$ and the points $M$ of a normal polygonal region $R_{\tau}$ lying in the half $\tau$-plane of Poincare and which is the transform of $R$ by means of a linear substitution. $R_{\tau}$ may be referred to as the space of the conjugate subgroups of the given properly discontinuous subgroup $H$ or simply the space of parameters or the functional space. It follows immediately that (i) any two such spaces are topologically equivalent, that is, homeomorphic; (ii) the space $R_{\tau}$ is reduced if symmetric, for example, the functional space of the group $H$ generated by a zero-angled circular triangle $T$ inscribed to $|z|<1$ and its repeated symmetries with respect to the sides ad infinitum and of any of its subgroups $\bar{H} C H$ is the modular triangle $T_{r}$. (Received May 11, 1948.)

393t. Cengiz Uluçay: On the values of the Bloch-Landau constants $\mathfrak{B}$, R. II.

Fundamental Lemma II. A theorem of uniqueness. Let $\rho w=f(z ; M)$, where $|\rho|$ is a 
positive scale constant, be the analytic function of modular parabolic type which maps a zero-angled circular triangle $T$ conformally on to a straight equilateral triangle $\left(T_{w}\right)_{\mathrm{I}}$. Normalizing the above function and calling $\left(L_{M}\right)_{\mathrm{I}}$ the radius of the "assumed" circle circumscribed to $\left(T_{w}\right)_{\mathrm{I}}$ one has (i) clearly $\mathfrak{l} \leqq\left(L_{M}\right)_{\mathrm{r}}$, (ii) let $T_{\tau}$ be the space of parameters bounded by the two positive half rays $R_{\tau}=0, R_{\tau}=1$ and the upper semicircle $|2 \tau-1|=1$. The arcs of circle of radius unity centered at $\tau=0, \tau=1$ and the positive half ray $R_{\tau}=1 / 2$ intersect at $\mathfrak{F}=1 / 2+i 2^{(1 / 2)} / 2$. The theorem of uniqueness is that $\left(L_{\xi}\right)_{\mathrm{I}}<\left(L_{M}\right)_{\mathrm{I}}$ for all points $M$ which do not coincide with $\mathfrak{F}$. If instead of $\left(T_{w}\right)_{\text {I }}$ one would consider straight triangles $\left(T_{w}\right)_{\text {II }}$ and $\left(T_{w}\right)_{\text {III }}$ with the angles $\pi / 2$, $\pi / 4, \pi / 4$ and $\pi / 2, \pi / 3, \pi / 6$ respectively, then by Lemma I, (i) $\left(L_{\mathfrak{F}}\right)_{\mathrm{II}}<\left(L_{M}\right)_{\mathrm{II}}$, $\left(L_{\mathfrak{F}}\right)_{\mathrm{III}}<\left(L_{M}\right)_{\mathrm{III}}$ for all $M \neq \mathfrak{F}$, (ii) $\left(L_{\mathfrak{F}}\right)_{\mathrm{I}}=0.54326<\left(L_{\mathfrak{F}}\right)_{\mathrm{II}}<\left(L_{\mathfrak{F}}\right)_{\mathrm{III}}$ (this estimate has been first obtained by Rademacher: On the Bloch-Landau constant, Amer. J. Math. (1943)). (Received May 11, 1948.)

\section{4t. Cengiz Uluçay: On the values of the Bloch-Landau constants} B, R. III.

Let $T^{l, m, n}$, where the superscripts shall be dropped whenever $l=m=n=1$, be a circular triangle lying in $|z|<1$ and bounded by circular arcs orthogonal to $|z|=1$. Let $\pi / 6 l, \pi / 6 m, \pi / 6 n$, where $l, m, n$ are positive integers subject to the condition $1 / l+1 / m$ $+1 / n<6$, denote the angles of the circular triangle. Let $\rho w=f^{l, m, n}(z ; M)$ be the analytic function of elliptic type which maps $T^{l, m, n}$ conformally onto $\left(T_{w}\right)_{\mathrm{I}}$. By Lemma I one may assume $M$ generating the functional space $T_{\tau}$. Then (i) clearly $\mathfrak{B} \leqq\left(B_{M}^{l, m, n}\right)_{\mathrm{r}}$, (ii) every point $M$ determines uniquely $B_{M}^{l, m, n}$ for every set of integers $l, m, n$ and such that $\left(B_{M}\right)_{I} \leqq\left(B_{M}^{l, m, n}\right)_{I}$ where the equality holds if and only if $l=m=n=1$ (the proof of this theorem requires the use of Schwarz' lemma). Moreover the correspondence between $\left(B_{M}\right)_{\mathrm{I}}$ and $\left(B_{M}^{l, m, n}\right)_{\mathrm{I}}$ is monotonic increasing (by the use of Lemma I and Lemma II). Consequently $\left(B_{\mathfrak{F}}\right)_{\mathrm{I}}<\left(B_{M}\right)_{\mathrm{I}}$ for all $M \neq \mathfrak{F}$. Similar considerations hold in the case of $\left(T_{w}\right)_{\mathrm{II}}$ and $\left(T_{w}\right)_{\mathrm{III}}$, whereby $(B \mathfrak{F})_{\mathrm{I}}=0.4719<(B \mathfrak{F})_{\mathrm{II}}<(B \Im)_{\mathrm{III}}$ (this estimate has been first obtained by Ahlfors and Grunsky: Über die Blochsche Konstante, Math. Zeit. (1936)). (Received May 11, 1948.)

\section{5t. Cengiz Uluçay: On the values of the Bloch-Landau constants} $\mathfrak{B}, \mathfrak{R}$. IV.

Consider the hexagon $T: G A G^{\prime} E B E^{\prime} F C F^{\prime}$ where $G^{\prime} E, E^{\prime} F, F^{\prime} G$ are equal arcs of the circle orthogonal to the unit circle $|z|=1$ whereas $G A G^{\prime}, E B E^{\prime}, F C F^{\prime}$ are equal arcs of the circle belonging to the circumference of $|z|<1$. Thus $T$ is symmetric with respect to the axes $O A, O B, O C$ which make with each other an angle of $2 \pi / 3$ and $T$ is circumscribed to the concentric circle of radius $s_{0}<s<1$ where $s_{0}$ is the radius of the circle inscribed in the zero-angled circular triangle $A B C$. Then $w=z+\cdots$ is such that it maps $T$ conformally onto a straight equilateral triangle $T_{w}$ : efg, with the cuts $g a, e b, f c$ issuing from the vertices $e, f, g$ and extending halfway along the radii $o g, o e$, of where $o$ is the centre of $T_{w}$. The length of these cuts uniquely determines $s$ which need not be calculated explicitly (in any case it is not difficult to determine a priori the corresponding value of $s$, in fact it is $s=3^{1 / 2} / 3$ ). It is clear that $w=z+\cdots$ cannot "assume" a circle of size greater than the circle inscribed in $T_{w}$. If $\mathfrak{Q}^{*}$ denotes the radius of this circle than it is found that $\mathfrak{R}^{*}=0.64$. If $\rho w=f(z ; M)$ is the family of analytic functions of hyperbolic type regular in $|z|<1$ and such that $w=z+\cdots$ is contained in it then $\mathfrak{l}^{*}$ is again uniquely determined within the family. (Received May 11, 1948.) 
396t. Cengiz Uluçay: On the values of the Bloch-Landau constants $\mathfrak{B}, \mathfrak{R} . \mathrm{V}$.

Let $w=f(z)=z+\cdots$ be a Bloch function $\mathbb{E}$ where $\mathfrak{E}$ stands for $\mathfrak{B}$ or $\mathbb{\&}$ according to cases (R. M. Robinson: Bloch functions, Duke Math. J. (1936)). As usual let $\rho w=f(z ; M)$ be the family of analytic functions regular in $|z|<1$ and containing $w=f(z)$. To each function of the family is associated its group $H^{\prime}=g \mathrm{Hg}^{-1}, g \in H$. The totality of these functions can thus be represented by points $M$ in the functional space $R_{\tau}$ which is quasi-compact or compact according as $M$ can or cannot reach the real axis. As $M$ varies continuously in $R_{\tau}$ the open circle "assumed univalently" or "assumed", according to cases, by the corresponding normalized function varies also continuously. By Lemma I and II the correspondence between the size of these circles and the size of the circles $\left(L_{M}\right)_{\mathrm{I}}$ is monotonic increasing. With the help of this uniqueness theorem it follows readily that $H$ is derived from a fundamental triangle and its repeated symmetries ad infinitum. Hence $\mathfrak{B}=(B \mathfrak{F})_{\mathrm{r}}, \mathfrak{l}=\left(L_{\mathfrak{F}}\right)_{\mathrm{r}}<\mathbb{R}^{*}$ as conjectured by Ahlfors-Grunsky and Rademacher respectively. This last step presupposes essentially that $H$ does not reduce to the identity element which is the case. Hence it fails in the case of a Bloch function $\mathfrak{U}$ since the latter is univalent in $|z|<1$. Finally it is conjectured that $\mathfrak{u} \geqq \mathfrak{R}^{*}$. (Received May 11, 1948.)

\section{7t. J. G. Wendel: A degenerate van der Pol equation. Preliminary report.}

The equation (1): $k \dot{x}+f(x) \dot{x}+k g(x)=e(t), k$ small and positive, is considered, relative to the degenerate equation $(2): f(y) \dot{y}=e(t) . F(x)$ and $E(t)$ denote fixed indefinite integrals of $f$ and $e$ respectively; $F(x) \operatorname{sgn} x \rightarrow \infty$ as $|x| \rightarrow \infty$. If $F$ and $E$ are piecewise strictly monotone then solutions of (2) can be defined, which exist for all $t \geqq t_{0}$, but which are in general discontinuous. The two chief results are I: Solutions $x(t)$ of (1) for which $x\left(t_{0}\right)=x_{0}, f\left(x_{0}\right)>0,\left|\dot{x}\left(t_{0}\right)\right| \leqq k^{-1 / 2}$, tend as $k \rightarrow 0$ to the solution $y\left(t ; x_{0}, t_{0}\right)$ of $(2)$ for which $y\left(t_{0}\right)=x_{0}$; the convergence is uniform in $x_{0}$ and $t$ if $t_{0} \leqq t$ $\leqq t_{0}+T$ and the discontinuities of $y(t)$ have been isolated. II: If $E(t)$ is periodic with period $p$; if $g(x)$ is strictly monotone increasing; if $x_{0}$ and $t_{0}$ exist such that $y\left(t ; x_{0}, t_{0}\right)$ has period $p, g\left(y\left(t ; x_{0}, t_{0}\right)\right)$ has mean value zero, and $F(x)=F\left(x_{0}\right)$ implies $x=x_{0}$-then for any $\delta$ there exists a $k_{0}$ such that for $0<k \leqq k_{0}$ any solution $x(t)$ of (1) satisfies $\left|x\left(t_{0}+n p\right)-x_{0}\right| \leqq \delta$ for all sufficiently large $n$. In the equation of Cartwright and Littlewood $k \ddot{x}+\left(x^{2}-1\right) \dot{x}+k x=b \lambda \cos (\lambda t+\alpha)$ (J. London Math. Soc. vol. 20 (1945) pp. 180-189) the condition $b>2 / 3$ is necessary and sufficient for the hypotheses of II to be satisfied. (Received April 27, 1948.)

\section{Applied Mathematics}

398. J. P. Ballantine: The electrical resistance of certain infinite nets.

$N$ is an infinite screen each segment of which has a resistance of $1 \mathrm{ohm} . P$ and $Q$ are two certain vertices of $N$. The net resistance between $P$ and $Q$ is then found. In another problem, $N$ has infinite dimensions one way, and finite dimensions the other. When the finite dimension is limited to 8 segments, the computation of the resistance between $P$ and $Q$ is reduced to the solution of 4 quadratic equations, and 4 simultaneous linear equations in 4 unknowns. (Received May 10,1948.)

399. Gertrude Blanch: On the normalization of Mathieu functions. 
The solutions of period $\pi$ and $2 \pi$ of Mathieu's differential equation $y^{\prime \prime}+\left(b-s \cos ^{2} x\right) y$ $=0$ have been widely studied. Once the characteristic values $b$, corresponding to the given parameter $s$, have been obtained, these solutions are completely determined, apart from an arbitrary constant factor which can be fixed by suitably normalizing the solutions. Two normalizations are in current use: (1) The normalization determined by setting $y(0)=1$ or $y^{\prime}(0)=1$, depending on whether the periodic solution is different from zero or not, at $x=0$. (2) The normalization determined by setting the integral from zero to $2 \pi$ of $y^{2}$, with respect to $x$, equal to $\pi$. It is shown that although the normalization (2) has one desirable property, namely that the solution ranges numerically between 0 and 10 practically everywhere, these solutions cannot now be readily determined for large values of $s$, when the trigonometric series becomes too cumbersome to use. Moreover, the proponents of the normalization (2) have failed to point out that for large $s$, the desirable range of the solution does not necessarily give any useful information about the order of magnitude of the solutions. In contrast to this, the solutions as normalized according to (1) can all be determined asymptotically with relative ease when $s$ is large, and their order of magnitude can be obtained everywhere from the asymptotic expansions. It would therefore seem that with the current knowledge about the asymptotic developments for these solutions, the normalization (1) is the better one to adopt. (Received May 13, 1948.)

400. Albert Cahn: Some formulae for representing $\int_{0}^{x} J_{n}(t) d t$ and $\int_{0}^{x} Y_{n}(t) d t$.

From elementary properties of Bessel functions follow the relationships: $\int_{x}^{\infty} C_{\nu} t^{-q} d t=C_{\nu-1} x^{-q}+(n-q-1) \int_{x}^{\infty} C_{\nu-1} t^{-(q+1)} d t ; \quad \int_{x}^{\infty} C_{\nu} t^{-q} d t=(n+q-1)^{-1}\left[C_{\nu} x^{-(q-1)}\right.$ $\left.+\int_{x}^{\infty} C_{\nu-1} t^{-(q-1)} d t\right]$; where $C_{\nu}$ represents either $J_{\nu}$ or $Y_{\nu}$. These equations may be used to obtain a representation of $\int_{x}^{\infty} C_{n}(t) d t$ in terms of Bessel functions of order less than $n$ and the integral $\int_{0}^{x} C_{0}(t) d t$. Such representations are useful in checking the results of numerical integrations of Bessel functions. (Received May 15, 1948.)

\section{Edmund Pinney: The floating elastic sphere.}

The stresses and displacements are obtained for an isotropic elastic sphere floating in a fluid and acted upon by the force of gravity. In the particular case in which the sphere is completely submerged, the answer is in closed form. In this case the sphere is compressed and slightly flattened in the vertical direction. (Received May 17, 1948.)

402. G. M. Volkoff and D. S. Carter: On the kinetic theory of the viscosity of gases.

The traditional theory of Maxwell for the transport of momentum in a gas as usually presented in standard texts (cf. Page, Introduction to theoretical physics) fails to account for the fact that the stress tensor is symmetric. An alternative elementary kinetic theory derivation of the coefficient of viscosity is given which yields the usual value and, at the same time, demonstrates by a purely geometric argument that the stress tensor is symmetric. (Received May 11, 1948.)

\section{GeOMETRY}

403t. W. L. Chow: On the genus of curves of an algebraic system.

A simple, purely algebraic proof is given for the well known theorem that the 
genus of any curve of an algebraic system of algebraic curves is not greater than the genus of the generic curve of the system. The method is based on the idea of the associated form of an algebraic variety, which was introduced by van der Waerden and the author some years ago. (Received May 10, 1948.)

\section{4t. David Ellis: Superposability properties of metric groups.}

Let $G$ be an additively written Abelian group and denote by $|G|$ the set of pairs $(a,-a)$ of inverse elements of $G$. A metric group in the sense of Menger is obtained by attaching to each order pair $a, b$ of elements of $G$ the element $(b-a, a-b)$ of $|G|$ as distance. In the paper introducing this notion (Math. Zeit. vol. 33 (1931) pp. 396418) Menger showed that a metric group has two-point superposability; that is, corresponding to each two congruent pairs of elements of $G$ there is a congruence of $G$ with itself that maps one pair of elements onto the other. In this note two-point superposability is given a two-fold extension. It is shown that (1) any two congruent subsets of a metric group are superposable, and (2) any congruence between any two subsets is extendable to the entire group. (Received March 30,1948.)

\section{J. W. Green: On the centroids of convex areas.}

The region of variation of the centroid of a convex area in the plane is computed, where the convex area is subjected to certain conditions; for example, if the area is required to contain a line segment as boundary and to have prescribed supporting lines at the end of the segment. Several applications are made, and the results include the following: If lines are drawn through the centroid of a convex area dividing it in two areas, the locus of the centroids of the areas is a convex curve. (Received May 15, 1948.)

\section{6t. C. C. Hsiung: Rectilinear congruences.}

In his prize memoir (Sur la thérie gênérale des congruences, Mémoires publiés par la classes des sciences de l'Académie Royale de Belgique (2) vol. 3 (1911)) Wilczynski has established the theory of a rectilinear congruence in ordinary three-dimensional projective space by using a system of linear partial differential equations. However, his method of deriving the system of the differential equations is not completely geometric. The author proposes to remedy this lack of geometric content in the present paper. By purely geometric determinations, a completely integrable system of linear homogeneous partial differential equations in canonical form is introduced, defining a rectilinear congruence $\mathcal{L}$ in ordinary space except for a projective transformation. Some invariants and covariants of these equations and a completely invariant coordinate system are obtained. The local power series expansions for the two focal surfaces and the curves of the two focal nets of the congruence $\mathcal{L}$ are calculated and expressed in terms of the invariants. The author also studies some covariant congruences associated with the congruence $\mathcal{L}$ and presents simple geometric characterizations of $W$ congruences, Segre-Darboux congruences and some other special congruences. (Received May 14, 1948.)

407. Peter Scherk: On the minimal spaces of curves of order $n+1$ in projective $n$-space. Preliminary report.

A $K^{n+1}$ is a differentiable closed curve in real projective $n$-space that has a maximum number of $n+1$ points in common with any hyperplane. If an $m$-space is spanned 
by osculating spaces it is called normal [abundant] if it meets the $K^{n+1}(m+1)$ times $[(m+2)$ times]. A minimal space is an abundant space that contains no other abundant subspaces. The following results are proved: (1) Each $m$-space contains at most one minimal subspace. A space spanned by osculating spaces contains a minimal subspace if and only if it is abundant $[m<n]$. (2) Suppose the $m$-space $E$ is normal and the point $s$ lies on the $K^{n+1}$ but not on $E$. Then the projection of $s$ from $E$ is $q$-times singular if and only if either $s$ is $(q+m+1)$-times singular or if $E$ meets the osculating $(n-m-q)$-space but not the osculating $(n-m-q-1)$-space of $s[n \geqq 2 ; 0 \leqq m \leqq n-2$; $1 \leqq q \leqq n-m-1]$. (Received May 10, 1948.)

\section{8t. Peter Scherk: On surfaces of constant curvature.}

By a well known theorem, the Gaussian curvature of a surface is constant if all the geodesic distance circles on the surface have constant geodesic curvatures (cf., for example, Blaschke: Vorlesungen iber Differentialgeometrie, vol. 1, 3d ed., 1930, pp. 154-155). This theorem is a corollary of the following observation: Suppose that the geodesic distance circles about one point have constant geodesic curvatures. Then the Gaussian curvature at a point on these circles depends only on its geodesic distance from the first point. So the following stronger result may be stated: Suppose there exists a finite or infinite set of points on the surface so that the geodesic curvatures of the geodesic distance circles about these points are constant, and suppose any two points of the surface can be joined by a polygon whose sides are arcs of geodesic distance circles about points of the set. Then every geodesic distance circle has constant geodesic curvature and the Gaussian curvature of the surface is constant. (Received May 10, 1948.)

409t. Peter Scherk: On the minimal osculating spaces of curves of order $n+1$ in projective $n$-space. I. Preliminary report.

The minimal spaces of a closed curve $K^{n+1}$ of order $n+1$ in real projective $n$-space are defined in abstract 54-9-407. Let $N_{p_{1}}^{n} \ldots, p_{j}$ denote the number of minimal spaces through $j$ different points of the curve that contain the osculating $p_{1}$-space of one of them, the osculating $p_{2}$-space of another, etc. Put $A_{n}=\sum_{0}^{n-1}(n-p) N_{p}^{n}$, $B_{n}=\sum_{1}^{n-2}(n-p-1) N_{p 0}^{n}$. The author conjectures $A_{n}+B_{n} \leqq n+1$. He proved before $A_{n} \leqq n+1 ; A_{n}+B_{n}=O\left(n^{2}\right)$ [Ann. of Math. vol. 46 (1945) pp. 68-82, 175-181]. He

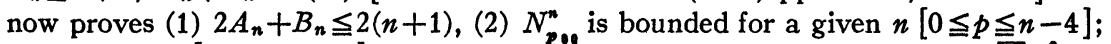
$N_{p_{10}}^{n}$ is finite $[0 \leqq p \leqq n-5]$. (3) If $N_{p_{0}}^{n}=0$ for $p \neq n(\bmod 2)$, then $N_{n-8,1}^{n}=\sum_{2}^{n-2} N_{p_{0}}^{n}$ $\left[{ }_{n}>4\right]$. (4) The $K^{n+1}$ with $N_{10}^{n}+N_{000}^{n}>0$ are classified. (Received May 10, 1948.)

\section{LoGic AND Foundations}

\section{0t. A. P. Morse: Squares are normal.}

Two plane sets are finitely equivalent if and only if they can be split respectively into sets $a_{1}, a_{2}, \cdots, a_{n}$ and into sets $a_{1}^{\prime}, a_{2}^{\prime}, \cdots, a_{n}^{\prime}$ in such a way that the corresponding subdivisions are congruent. A plane set $S$ is paradoxical if and only if it can be split into two sets each of which is finitely equivalent to $S$. A plane set which is not paradoxical is normal. It has been known for some time that squares and a variety of other sets are normal. However, all known verifications of the normality of squares depend in an essential way on the axiom of choice. In the present paper, which will appear in Fundamenta Mathematicae, it is found that it is indeed possible to show, without the axiom of choice, that any bounded plane set with inner points is normal. (Received April 21, 1948.) 


\section{Statistics AND Probability}

411t. Waclaw Kozakiewicz: On the necessary and sufficient conditions for the convergence of a sequence of moment generating functions. Preliminary report.

Let $\left\{F_{n}\left(x_{1}, x_{2}\right)\right\}$ be a sequence of distribution functions, and let $\left\{\phi_{n}\left(t_{1}, t_{2}\right)\right\}$ be the corresponding sequence of moment generating functions all of which are assumed to exist for $\left|t_{i}\right|<\alpha_{i}, \alpha_{i}>0 \quad(i=1,2)$. Assuming $z_{i} \geqq 0 \quad(i=1,2)$, let $M_{n}\left(z_{1}, z_{2}\right)$ $=\iint_{R} d F_{n}\left(x_{1}, x_{2}\right)$, where $R$ is the region (dependent on $\left.z_{1}, z_{2}\right)$ for which the inequalities $\left|x_{i}\right| \geqq z_{i}(i=1,2)$ are satisfied. Let $M\left(z_{1}, z_{2}\right)$ be the least upper bound of the sequence $\left\{M_{n}\left(z_{1}, z_{2}\right)\right\}$. It may be shown that the necessary and sufficient conditions for the convergence of the sequence $\left\{\phi_{n}\left(t_{1}, t_{2}\right)\right\}$ for $\left|t_{i}\right|<\alpha_{i}(i=1,2)$ are: (a) for any fixed $t_{i}$ satisfying the inequality $\left|t_{i}\right|<\alpha_{i}(i=1,2)$, there exists a number $K$ (dependent only on $t_{1}$ and $\left.t_{2}\right)$ such that $M\left(z_{1}, z_{2}\right) \leqq K \exp \left(-\left|t_{1}\right| z_{1}-\left|t_{2}\right| z_{2}\right)$ for all $z_{i} \geqq 0(i=1,2)$; (b) $\left\{F_{n}\left(x_{1}, x_{2}\right)\right\}$ converges to a distribution function $F\left(x_{1}, x_{2}\right)$ at each point of continuity of $F\left(x_{1}, x_{2}\right)$. Further, it may be proved that when the conditions (a) and (b) are satisfied, the moment generating function of $F\left(x_{1}, x_{2}\right)$ exists for $\left|t_{i}\right|\left\langle\alpha_{i}(i=1,2)\right.$, and is equal to the limit of the sequence $\left\{\phi_{n}\left(t_{1}, t_{2}\right)\right\}$ for $\left|t_{i}\right|<\alpha_{i}(i=1,2)$. All results can be easily extended to the case when distribution functions and moment generating functions are defined in the Euclidean space of any finite number of dimensions. (Received May 14, 1948.)

\section{TOPOLOGY}

\section{2t. R. D. Anderson: Concerning upper semi-continuous collec- tions of continua.}

The author proves that if $M$ is any compact continuous curve and $\epsilon$ is any positive number, there exists a finite collection $G$ of continuous curves filling up $M$ each of diameter less than $\epsilon$ such that if $H$ is any subcollection of $G$ the common part of all the continua of $H$, if it exists, is the sum of a finite number of continuous curves. With the aid of this result, he shows that if $M$ is any locally compact completely separable metric continuous curve there exist a one-dimensional continuous curve $K$ in threedimensional Euclidean space and an upper semi-continuous collection of mutually exclusive compact continua filling up $K$ which with respect to its elements as points is topologically equivalent to $M$. (Received May 4, 1948.)

\section{A. L. Blakers: Obstructions to deformations of mappings.}

Let $K$ be a simplicial complex, $X$ and $A$ arcwise connected topological spaces, with $A \subset X$. Assume also that the fundamental group $\pi_{1}(A)$ operates simply on the relative homotopy group $\pi_{n}(X, A)$. Let $f$ be a mapping $f: K \rightarrow X$ which maps the $n$-skeleton $K^{(n)}$ into $A$. Let $\sigma^{n+1}$ be an oriented $(n+1)$-simplex of $K$. Then the mapping $f \mid \sigma^{n+1}:\left(\sigma^{n+1}, \sigma^{n+1}\right) \rightarrow(X, A)$ determines uniquely an element $\alpha \in \pi_{n+1}(X, A)$, and the function defined by $c^{n+1}\left(f ; \pi_{n+1}(X, A)\right)\left(\sigma^{n+1}\right)=\alpha$ is an $(n+1)$-cochain of $K$. with coefficients in $\pi_{n+1}(X, A)$. It is shown by homotopy addition theorems that $c^{n+1}\left(f ; \pi_{n+1}(X, A)\right)$ is a cocycle and is trivial if and only if the mapping $f$ can be deformed by a homotopy which is constant over $K^{(n)}$ to a mapping which takes $K^{(n+1)}$ into $A$. It is also shown that $c^{n+1}\left(f ; \pi_{n+1}(X, A)\right)$ is a coboundary if and only if $f$ can be deformed by a homotopy which is constant over $K^{(n-1)}$ to a mapping which takes $K^{(n+1)}$ into $A$. Thus the cocycle $c^{n+1}\left(f ; \pi_{n+1}(X, A)\right)$ is related to deformations of map- 
pings in the same way that the obstruction cocycle of Eilenberg (Ann. of Math. vol.41 (1940) pp. 231-251) is related to extensions of mappings. (Received April 27, 1948.)

\title{
414. S. P. Diliberto: Poincarê's geometric theorem. Preliminary
} report.

The writer gives a new proof of Poincare's geometric theorem which simplifies Birkhoff's proof (An extension..., Acta Math. vol. 47 (1926)), and which removes the requirement that one boundary be a circle and the other star shaped: If $A$ is a closed annular region bounded by a pair of nested, simple, Jordan curves $C_{i}(i=1,2)$ and $T$ an area preserving homeomorphism of $A$ onto itself, the $C_{i}$ having rotation numbers of opposite sign, then $T$ must have at least two distinct fixed points. $A$ is replaced by $A^{*}$, its universal covering space: an infinite strip bounded by a pair of parallel lines, and $T$ by a periodic map $T^{*}$ of $A^{*}$ onto itself. The mapping vectors of $T^{*}$ are shown to have a singular point of index one by a device using Brouwer's translation arcs (Kerekjarto, Acta Univ. Szeged. vol. 4 (1928)). The second fixed point follows from the index sum theorem. (Received May 17, 1948.)

\section{5t. M. H. A. Newman: Local connection in locally compact spaces.}

It was proved by Hurewicz in 1935 (Fund. Math. vol. 25 (1935) pp. 467-485) that a compact space which is both $L^{1}$ and $l^{n}$ is $L^{n}$. In the present paper the corresponding result for locally compact spaces is proved, (a) for uniform local connection, and (b) for relative local connection, thereby settling affirmatively a question posed by Eilenberg and Wilder (Amer. J. Math. vol. 64 (1942) pp. 613-622). The main lemma used by Hurewicz, on the passage from $\epsilon$-homotopy to true homotopy, cannot be carried over to locally compact spaces without substantial modification. A stronger form of the $\mathrm{lc}^{p}$ and $\mathrm{LC}^{p}$ conditions is therefore used, namely (for $\mathrm{lc}^{p}$ ) the existence of a function $\phi(\delta, x)$ such that, given a compact set $F$ in the neighborhood $U(x, \phi)(\delta, x))$ of any point $x$, there is a compact subset $F^{\prime}$ of $U(x, \delta)$ such that every $q$-cycle in $F$ bounds in $F^{\prime}$, for $0 \leqq q \leqq p$; and analogously for LC ${ }^{p}$. These conditions are proved equivalent to the usual definitions in locally compact (metric) spaces. (Received May 12, 1948.)

\author{
J. W. GReEN, \\ Associate Secretary
}

